\title{
In and Out of Consciousness: How Does Conscious Processing (D)evolve Over Time?
}

\author{
Jaan Aru ${ }^{1,2 *}$ and Talis Bachmann ${ }^{1}$ \\ ${ }^{1}$ Department of Penal Law, School of Law, University of Tartu, Tartu, Estonia, ${ }^{2}$ Chair of Data Science, Institute of Computer \\ Science, University of Tartu, Tartu, Estonia
}

Keywords: consciousness, NCC, microgenesis, time course of consciousness, transitions of consciousness

\section{OPEN ACCESS}

Edited by:

Morten Overgaard,

Aarhus University, Denmark

Reviewed by:

Giorgio Marchetti,

Mind, Consciousness, and Language Research Net, Italy

*Correspondence:

Jaan Aru

jaan.aru@gmail.com

Specialty section:

This article was submitted to Consciousness Research, a section of the journal Frontiers in Psychology

Received: 25 October 2016 Accepted: 18 January 2017 Published: 02 February 2017

Citation:

Aru J and Bachmann T (2017) In and Out of Consciousness: How Does Conscious Processing (D)evolve Over

Time? Front. Psychol. 8:128. doi: 10.3389/fpsyg.2017.00128
Mental information processing includes both unconscious and conscious modes and there are transitions between these two. The content of subjective experience can emerge from preconscious content, but an opposite process of loss of conscious content or its decay from subjective experience is an inevitable reality as well. Both ways of transition on the border between unconscious and conscious processing are ubiquitous. But how does this transition unfold over time? While there is quite some literature on whether conscious perception is all-or-none or graded (Sergent and Dehaene, 2004; Overgaard et al., 2006), we ask the complementary question: how does conscious perception change and evolve over time? While in phenomenological approaches in the philosophy of mind all-embracing temporal perspective has been acknowledged as crucial in understanding consciousness (e.g., anticipation, present, and retention in Husserl, 1928), in experimental paradigms only narrow temporal slices have been typically examined.

We will first focus on the knowledge about the transitions gained from studying brief visual stimuli. According to the microgenetic tradition mental content does not emerge instantaneously, in an all-or-none manner (review: Bachmann, 2000). Instead, conscious content arises as a gradual process of formation where the initial transition (there was no content and now there is some content) grows over to a time consuming process where subjective phenomenal content of the same intentional object matures by acquiring systematically more qualities to the preceding version of the percept. This intentional object might be a visual object, scene, memory representation etc. These microgenetically developing attributes or characteristics include subjective clarity, subjective contrast, subjective fragmentariness/exhaustiveness, coarseness/detail, subjective stability, etc. (Bachmann, 2000, 2012). In other words, conscious experience of the content pertaining to the same intentional object changes considerably over time (See also Hegdé, 2008; Breitmeyer, 2014; Pitts et al., 2014).

On the other hand (especially when brief objects typical to most of the experiments are presented) a certain experience with its phenomenal subjective content sooner or later disappears from consciousness by an analogous, but reversed gradual process-a kind of "anti-genesis" (Bachmann, 2000). Figure 1 illustrates the notion of microgenesis with its formative and disformative stages. Note that this figure is an abstraction based on the empiricial research described in Bachmann (2000). However, we hope that provocatively drawing this time course inspires researchers to investigate how it exactly looks like.

This simple conceptualization prompts surprisingly many old and new questions.

1. What is the exact shape of this curve? How do changes in stimulus parameters (e.g., contrast, duration) change the shape?

2. Is there a kind of asymmetric inertia of formation and disformation as depicted on Figure 1? In other words, is it indeed so that disformation takes more time than formation of conscious content? To our mind, decay from consciousness seems to be slower mainly because psychophysical estimates of the speed of immediate perception by masking, temporal 


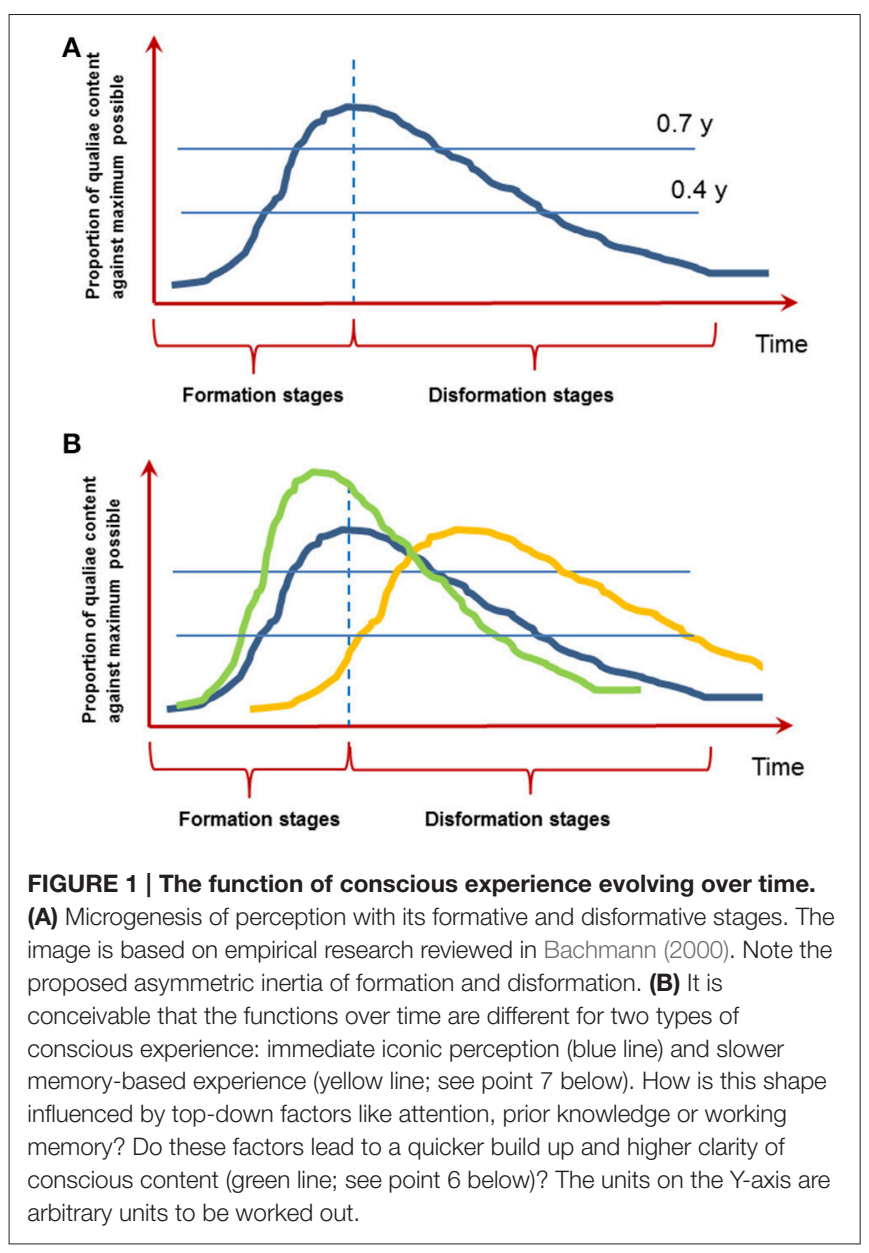

order estimation, speeded discrimination, and other experimental tasks are smaller than experimental estimates of the duration of immediate memory (Bachmann, 2000).

3. How to experimentally measure subjective content (a) at $\mathrm{y}_{\max },(\mathrm{b})$ at any optional stage of microgenesis (e.g., $\mathrm{y}=$ $0.4, y=0.7)$ ? In fact, there are many qualities of subjective evaluation unfolding in response to stimulation or task cue (e.g., Kalmus and Bachmann, 1980; Bachmann, 1980, 2012). The most recent successfully used subjective scales include the perceptual awareness scale (PAS) capitalizing on subjective clarity ratings by subjects as well as other methods (review: Timmermans and Cleeremans, 2015).

4. A corollary question is how subjective and objective measures of consciousness relate. Do they show a mutually similar formation and disformation curve over time?

5. Is there an analogous time function for unconscious processing of content as for conscious processing? If so, how do we disentangle the conscious and unconscious processes (Miller, 2007; Bachmann, 2009; Aru et al., 2012; De Graaf et al., 2012)? Note that for example Herzog et al. (2016) propose that conscious and unconscious processing have quite different temporal characteristics, which might turn out to be helpful in disentangling them.
6. How do attention, prior knowledge or working memory content influence the shape of this curve (Figure 1B)? For example we know that all of these factors speed up the entry into consciousness and in general enhance the clarity of conscious experience (e.g., Carrasco et al., 2004; Soto et al., 2010; Aru et al., 2016). So the curve depicted on Figure 1 should rise quicker and be higher (in terms on $y$ units). But what about the disformation stage-do all of these factors also change how quickly the content disappears from consciousness?

7. What is the typical time course of the formative and disformative microgenetic stages? Data from ERP and MEG research on neural correlates of consciousness suggest that the initial formative stage peaks at time delays around 150250 ms (reviewed in Bachmann, 1994; Koivisto and Revonsuo, 2010). However, P300 has also been frequently found to mark conscious experience (e.g., Sergent et al., 2005; Del Cul et al., 2007; Rutiku et al., 2015, 2016). Intriguingly, it is possible that there are two separate processes-(1) perceptual microgenesis, where conscious experience emerges fast and decays fast (possibly equal to iconic-memory decay) and (2) immediate memory-based microgenesis, where conscious experience of the same target forms a bit slower than perceptual microgenesis and decays much later than iconic delay (e.g., Sligte et al., 2008). It is even possible that while perceptual microgenetic function decays (disformation) the memory-based function is still building up (Figure 1B, yellow vs. blue line). This idea fits with the distinction between phenomenal and reflective consciousness, which are thought to depend on different types of attention (e.g., Koivisto et al., 2009).

Now, an intriguing theoretical question appears: if one and the same stimulus-event is related to both, perceptual and immediate-memory microgenetic processes with concomitant two sets of NCC, should we then regard these NCC as different aspects of one NCC or a principally different, two, NCCs (Bachmann, 2015)?

8. How does the time course of subjective microgenesis relate to the time course of representational content development obtained with neural decoding and representational similarity analysis (e.g., Carlson et al., 2013; Cichy et al., 2014; Goddard et al., 2016)?

9. What are the relative roles of feedforward and re-entrant neural processes in perceptual microgenesis? For example the "reverse-hierarchy" theory of Hochstein and Ahissar (2002) suggests that global features should emerge in consciousness faster than the local features. This crucial prediction was recently confirmed (Campana et al., 2016), leading to think that conscious perception might start at the highest levels of visual processing and acquire the fine details through feedback from higher to lower levels of visual processing.

10. Are the nature and regularities of microgenesis the same when an external stimulus is becoming microgenetically formed and when a memory-image of the same stimulus is evoked and formed? More generally: are the curves of formation and disformation similar for all the transitions occurring at the threshold of consciousness? There are many examples of 
transitions in and out of consciousness. Can one benefit from the knowledge gathered while studying brief visual stimuli to understand processes that unfold with other types of stimuli?

To understand this last question, let us list some examples about the transition into consciousness to illustrate the heterogeneity of this kind of transformation: remembering an item or idea as cued by external instruction or question; remembering an item or idea as ignited by intrinsic associative cues; having an insight; experience of an external sensory stimulus after its initial pre-conscious processing; experience of an already presented stimulus after focusing attention on it; becoming consciously aware of an intention (agency) to act after preconscious preprocessing of the action decision; becoming consciously aware of a different aspect (feature, attribute, property, quality) of a stimulus or scene after the preceding consciously aware experience of some other aspect(s); noticing the change in a change-blindness display; noticing the target in an inattentional blindness experiment; becoming consciously aware of the Gestalt content in Mooney face or Dalmatian dog types of image after an initially "meaningless" experience; becoming aware of the words within the sine-wave speech recording; reversal of binocularrivalry dominance in becoming aware of the suppressed stimulus; reappearance of the sensory afterimage. Is in all of these cases the emergence of conscious content gradually evolving over time?

There are also many examples for the transition out of consciousness: fading of the conscious percept; fading of iconic memory; loss of thought or imagery content; loss of explicitly experienced WM content; loss of conscious awareness of a stimulus after refocusing attention elsewhere; loss of a certain aspect (feature, attribute, quality) of conscious perception of a discontinued stimulation while other aspect(s) sustain; loss of conscious perception of a binocular-rivalry stimulus when it becomes suppressed; fading of perceptual content (e.g., color, spatial contrast modulation, luminance contrast step gradient) due to sensory adaptation; fading of afterimage. Is the fading of conscious content gradually evolving over time in all of these cases?

Gathering such a list leads yet again to interesting questions. For example, which transitions are reversible and which ones not? Loss of formed Gestalt content back to meaningless array of elements seems difficult; re-establishment of changeblindness/inattentional blindness seems impossible; after hearing the words within the sine-wave speech it is impossible to go back to hearing noise. What could this small set of non-reversibility tell us about the neural mechanisms of consciousness? The list

\section{REFERENCES}

Aru, J., Bachmann, T., Singer, W., and Melloni, L. (2012). Distilling the neural correlates of consciousness. Neurosci. Biobehav. Rev. 36, 737-746. doi: 10.1016/j.neubiorev.2011.12.003

Aru, J., Rutiku, R., Wibral, M., Singer, W., and Melloni, L. (2016). Early effects of previous experience on conscious perception. Neurosci. Conscious. 2016:niw004. doi: 10.1093/nc/niw004

Bachmann, T. (1980). Genesis of a subjective image. Acta et Commentationes Univ. Tartuensis. Prob. Cogn. Psychol. 522, 102-126. of these phenomena seems to suggest that conscious experience is heavily influenced by prior knowledge-once insightful knowledge about a particular stimulus is established, it is hard or even impossible to remove it.

More importantly, the variety of examples leads to the question whether there are general mechanisms and regularities underlying all of these phenomena. Do all these other types of transitions share some of the key features with the transitions happening in visual perception (Figure 1)? In visual perception it is relatively straightforward to "slice up" perception with techniques like visual masking (Bachmann, 1994; Bachmann and Francis, 2013), but even then studying the time course of visual perception is a time consuming and a difficult endeavor (Bachmann, 2000). Is it possible or even meaningful to try to do it with other types of transitions? How would one proceed with "slicing up" memory retrieval, Gestalt perception or insight formation? We do not have definitive experimental approaches, but we consider these questions to be important to put forth and to explore.

The present manuscript had a few aims: (1) we wanted to emphasize that conscious content evolves and changes over time, (2) we noted that the exact time course of how conscious content evolves over time is yet unknown and tentatively drew a time course to provoke more research in this direction, (3) we wanted to demonstrate that thinking about the time course of conscious processing prompts many interesting and intriguing questions, (4) finally we asked how general are such microgenetic regularities-do all kinds of transitions in and out of consciousness have gradual formation and disformation (Figure 1)? On the way we also seem to have stumbled on a few novel concepts applicable in studying dynamics of conscious experience-the formation/disformation (a)symmetry, reversibility, and the possibility of having two different NCCs for the same perceived object. We hope that some of these ideas and concepts are beneficial for unraveling the neural mechanisms of consciousness.

\section{AUTHOR CONTRIBUTIONS}

TB conceived the initial ideas, JA expanded them, both JA and TB discussed the ideas and contributed to writing the manuscript

\section{ACKNOWLEDGMENTS}

This work is partly supported by institutional Grant IUT20-40 from Estonian Ministry of Education and Research. Conscious Experience. Commack, NY: Nova Science Publishers.

Bachmann, T. (2000). Microgenetic Approach to the Conscious Mind. Amsterdam; Philadelphia: John Benjamins.

Bachmann, T. (2009). Finding ERP-signatures of target awareness: puzzle persists because of experimental co-variation of the objective and subjective variables. Conscious. Cogn. 18, 804-808. doi: 10.1016/j.concog.2009.02.011

Bachmann, T. (2012). How to begin to overcome the ambiguity present in differentiation between contents and levels of consciousness? Front. Psychol. 3:82. doi: $10.3389 /$ fpsyg. 2012.00082 
Bachmann, T. (2015). On the brain-imaging markers of neural correlates of consciousness. Front. Psychol. 6:868. doi: 10.3389/fpsyg.2015.00868

Bachmann, T., and Francis, G. (2013). Visual masking: Studying Perception, Attention, and Consciousness. San Diego, CA; Oxford: Elsevier; Academic Press.

Breitmeyer, B. G. (2014). The Visual (Un)conscious \& Its (Dis)contents. Oxford: OUP.

Campana, F., Rebollo, I., Urai, A., Wyart, V., and Tallon-Baudry, C. (2016). Conscious vision proceeds from global to local content in goal-directed tasks and spontaneous vision. J. Neurosci. 36, 5200-5213. doi: 10.1523/JNEUROSCI.3619-15.2016

Carlson, T., Tovar, D. A., Alink, A., and Kriegeskorte, N. (2013). Representational dynamics of object vision: the first 1000 ms. J. Vis. 13, 1. doi: 10.1167/13.10.1

Carrasco, M., Ling, S., and Read, S. (2004). Attention alters appearance. Nat. Neurosci. 7, 308-313. doi: 10.1038/nn1194

Cichy, R. M., Pantazis, D., and Oliva, A. (2014). Resolving human object recognition in space and time. Nat. Neurosci. 17, 455-462. doi: 10.1038/nn.3635

De Graaf, T. A., Hsieh, P. J., and Sack, A. T. (2012). The 'correlates' in neural correlates of consciousness. Neurosci. Biobehav. Rev. 36, 191-197. doi: 10.1016/j.neubiorev.2011.05.012

Del Cul, A., Baillet, S., and Dehaene, S. (2007). Brain dynamics underlying the nonlinear threshold for access to consciousness. PLoS Biol. 5:e260. doi: 10.1371/journal.pbio.0050260

Goddard, E., Carlson, T. A., Dermody, N., and Woolgar, A. (2016). Representational dynamics of object recognition: feedforward and feedback information flows. Neuroimage 128, 385-397 doi: 10.1016/j.neuroimage.2016.01.006

Hegdé, J. (2008). Time course of visual perception: coarse-to-fine processing and beyond. Prog. Neurobiol. 84, 405-439. doi: 10.1016/j.pneurobio.2007.09.001

Herzog, M. H., Kammer, T., and Scharnowski, F. (2016). Time slices: what is the duration of a percept? PLoS Biol. 14:e1002433. doi: 10.1371/journal.pbio.1002433

Hochstein, S., and Ahissar, M. (2002). View from the top: hierarchies and reverse hierarchies in the visual system. Neuron 36, 791-804. doi: 10.1016/S0896-6273(02)01091-7

Husserl, E. (1928). Phänomenologie des inneren Zeitbewusstseins. Jahrbuch für Philosophie und Phänomenologische Forschung 9, 367-498.

Kalmus, M., and Bachmann, T. (1980). Perceptual microgenesis of complex visual pattern: comparison of methods and possible implications for future studies. Acta et Commentationes Univ. Tartuensis Actual Prob. Indus. Psychol. 529, 135-159.

Koivisto, M., and Revonsuo, A. (2010). Event-related brain potential correlates of visual awareness. Neurosci. Biobehav. Rev. 34, 922-934. doi: 10.1016/j.neubiorev.2009.12.002

Koivisto, M., Kainulainen, P., and Revonsuo, A. (2009). The relationship between awareness and attention: evidence from ERP responses. Neuropsychologia 47, 2891-2899. doi: 10.1016/j.neuropsychologia.2009.06.016
Miller, S. M. (2007). On the correlation/constitution distinction problem (and other hard problems) in the scientific study of consciousness. Acta Neuropsychiatr. 19, 159-176. doi: 10.1111/j.1601-5215.2007. 00207.x

Overgaard, M., Rote, J., Mouridsen, K., and Ramsøy, T. Z. (2006). Is conscious perception gradual or dichotomous? A comparison of report methodologies during a visual task. Conscious. Cogn. 15, 700-708. doi: 10.1016/j.concog.2006.04.002

Pitts, M. A., Metzler, S., and Hillyard, S. A. (2014). Isolating neural correlates of conscious perception from neural correlates of reporting one's perception. Front. Psychol. 5:1078. doi: 10.3389/fpsyg.2014.01078

Rutiku, R., Aru, J., and Bachmann, T. (2016). General markers of conscious visual perception and their timing. Front. Hum. Neurosci. 10:23. doi: 10.3389/fnhum.2016.00023

Rutiku, R., Martin, M., Bachmann, T., and Aru, J. (2015). Does the P300 reflect conscious perception or its consequences? Neuroscience 298, 180-189. doi: 10.1016/j.neuroscience.2015.04.029

Sergent, C., and Dehaene, S. (2004). Is consciousness a gradual phenomenon? Evidence for an all-or-none bifurcation during the attentional blink. Psychol. Sci. 15, 720-728. doi: 10.1111/j.0956-7976.2004.00748.x

Sergent, C., Baillet, S., and Dehaene, S. (2005). Timing of the brain events underlying access to consciousness during the attentional blink. Nat. Neurosci. 8, 1391-1400. doi: 10.1038/nn1549

Sligte, I. G., Scholte, H. S., and Lamme, V. A. (2008). Are there multiple visual short-term memory stores? PLoS ONE 3:e1699. doi: 10.1371/journal.pone.0001699

Soto, D., Wriglesworth, A., Bahrami-Balani, A., and Humphreys, G. W. (2010). Working memory enhances visual perception: evidence from signal detection analysis. J. Exp. Psychol. Learn. Mem. Cogn. 36, 441. doi: 10.1037/a0 018686

Timmermans, B., and Cleeremans, A. (2015). "Chapter 3: How can we measure awareness? An overview of current methods," in Behavioral Methods in Consciousness Research, ed M. Overgaard (Oxford: OUP), 21-46.

Conflict of Interest Statement: The authors declare that the research was conducted in the absence of any commercial or financial relationships that could be construed as a potential conflict of interest.

Copyright (c) 2017 Aru and Bachmann. This is an open-access article distributed under the terms of the Creative Commons Attribution License (CC BY). The use, distribution or reproduction in other forums is permitted, provided the original author(s) or licensor are credited and that the original publication in this journal is cited, in accordance with accepted academic practice. No use, distribution or reproduction is permitted which does not comply with these terms. 